Ergod. Th. \& Dynam. Sys. (1988), 8, 191-204

Printed in Great Britain

\title{
Some compact invariant sets for hyperbolic linear automorphisms of torii
}

\author{
ALBERT FATHI† \\ Department of Mathematics, Walker Hall, University of Florida, Gainesville, \\ Florida 32611, USA
}

(Received 19 January 1987 and revised 1 April 1987)

\begin{abstract}
If the action induced by a pseudo-Anosov map on the first homology group is hyperbolic, it is possible, by a theorem of Franks, to find a compact invariant set for the toral automorphism associated with this action. If the stable and unstable foliations of the Pseudo-Anosov map are orientable, we show that the invariant set is a finite union of topological 2-discs. Using some ideas of Urbański, it is possible to prove that the lower capacity of the associated compact invariant set is $>2$; in particular, the invariant set is fractal. When the dilatation coefficient is a Pisot number, we can compute the Hausdorff dimension of the compact invariant set.
\end{abstract}

At the end of the sixties Hirsch [Hi] asked what kind of compact sets can be invariant under a toral hyperbolic linear automorphism. Several people worked on the question, see [Bo], [Fra2], [Ha], [Ir1], [Ir2], [Ma1], [Ma2], [Pr], [Ur]. Essentially, the following facts are obtained in these papers:

(i) there exists compact invariant sets of arbitrary topological dimension [Ha], $[\mathbf{P r}]$;

(ii) if a compact invariant set contains a Lipschitz arc, then it must contain a subtorus which is invariant under some power of the linear map [Fra2], [Ma1];

(iii) an invariant $C^{1}$ submanifold must be a union of subtorii [Ma2].

One of the questions that remain is: can a hyperbolic toral automorphism have a compact invariant set which is an embedded submanifold different from a subtorus? The simplest submanifolds would be surfaces of genus $\geq 2$.

In this work, we will not answer this question but we will obtain, from pseudoAnosov maps, some invariant sets which are in fact images under finite to one maps from surfaces of higher genus; moreover, these maps are locally injective except at a finite set of points. By the results of Franks and Mañé, these sets must be 'unsmooth'. We show this by computing some lower bound on the capacity. In some cases, it is possible to compute the Hausdorff dimension of the invariant set.

In March 1987, David Fried told me that he knew theorem 5.2 and that it is a consequence of Shub's paper [S]; moreover, the definition we give of topological closed 1-forms is almost that of the Alexander cocycle which is used in [S]. For completeness, let us add that several years ago John Franks proved (unpublished) that a pseudo-Anosov map with orientable foliations and quadratic dilatation

† Address for reprints: CNRS UA 1169, Université Paris-Sud, France. 
coefficient is a ramified cover of a linear hyperbolic map of $\mathbb{T}^{2}$, this is, of course, covered by theorem 5.2 .

\section{A topological definition of closed complex 1-forms}

If $Z$ is a topological space, we denote by $\mathscr{C}(Z)$ the space of continuous complex valued functions on $Z$. This is a complex vector space. We denote by $C^{n}(Z)$ the group of singular $n$-cochains on $Z$, and by $d$ the coboundary operator on that complex.

Let $X$ be a compact space. Denote by $\mathscr{P}(X)$ the space of (continuous) paths in $X$ endowed with the compact open topology. Denote by $\mathscr{C}^{1}(X)$ the space $\mathscr{C}(\mathscr{P}(X))$. The vector space $\mathscr{C}^{1}(X)$ is a subvector space of the space $C^{1}(X)$ of singular complex-valued 1-cochains. Define the subspace $\mathscr{Z}^{1}(X)$ of $\mathscr{C}^{\prime}(X)$ consisting of 1 -cocycles. If $X$ is for example a manifold - or more generally a locally contractible space - the intersection $\mathscr{C}^{1}(X) \cap d C^{0}(X)$ is precisely the set $\{d \varphi \mid \varphi \in \mathscr{C}(X)\}$. Moreover, if $\omega$ is a smooth closed 1 -form on a compact manifold $X$, we can define an element $\tilde{\omega} \in \mathscr{C}^{1}(X)$ in the following way: if $\alpha$ is a continuous path, in $X$ we can homotope it with fixed extremities to a smooth path $\tilde{\alpha}$, we put $\tilde{\omega}(\alpha)=\int_{\tilde{\alpha}} \omega$; since $\omega$ is closed it is easy to see that $\tilde{\omega}$ is well defined and contained in $\mathscr{Z}^{1}(X)$. From what we have said it is easy to obtain the following theorem:

THEOREM. Let $X$ be a compact manifold. The map $\omega \mapsto \tilde{\omega}$ induces an isomorphism between $H^{1}(X, \mathbb{C})$ and $\mathscr{Z}^{1}(X) / d(\mathscr{C}(X))$.

The advantage of the space $\mathscr{C}^{\prime}(X)$ over the space of smooth 1 -forms is that if $f: X \rightarrow Y$ is any continuous map, it induces a continuous map $f^{*}: \mathscr{C}^{1}(Y) \rightarrow \mathscr{C}^{1}(X)$. The map $f \mapsto f^{*}$ is of course functorial.

\section{Some linear algebra}

Let $A: \mathbb{Z}^{n} \tilde{\rightarrow} \mathbb{Z}^{n}$ be a linear isomorphism. Call its minimal polynomial $P_{A}$. Suppose we can write $P_{A}=Q_{1} Q_{2}$, where $Q_{1}$ and $Q_{2}$ have no common factor. Consider Im $Q_{1}(A) \subset \mathbb{Z}^{n}$, it is isomorphic to some $\mathbb{Z}^{k_{1}}$, and $A$ induces a linear isomorphism $A_{1}: \mathbb{Z}^{k_{1}} \rightarrow \mathbb{Z}^{k_{1}}$. One can see that the minimal polynomial of $A_{1}$ is precisely $Q_{2}$. Moreover, the dimension $k_{1}$ is also the dimension of $\operatorname{Ker} Q_{2}(A) \subset \mathbb{Z}^{n}$. One can see this last fact in the following way: first $\operatorname{Im} Q_{1}(A) \subset \operatorname{Ker} Q_{2}(A)$ and $\operatorname{Im} Q_{2}(A) \subset$ $\operatorname{Ker} Q_{1}(A)$; secondly, since $Q_{1}$ and $Q_{2}$ have no common factor, Bezout's theorem shows that $\operatorname{Ker} Q_{1}(A) \cap \operatorname{Ker} Q_{2}(A)=\{0\}$ and

$$
\operatorname{Im} Q_{1}(A) \otimes \mathbb{Q} \oplus \operatorname{Im} Q_{2}(A) \otimes \mathbb{Q}=\mathbb{Z}^{n} \otimes \mathbb{Q}=\mathbb{Q}^{n} .
$$

We can sum up what we have obtained in the following theorem.

THEOREM 2.1. Let $A: \mathbb{Z}^{n} \underset{\sim}{ } \mathbb{Z}^{n}$ be a linear isomorphism whose minimal polynomial $P_{A}$ can be written $P_{A}=Q_{1} Q_{2}$, where $Q_{1}$ and $Q_{2}$ have no common factor. Then, we have:

(i) the dimension $k_{1}$ of the image $\operatorname{Im} Q_{1}(A)$ is equal to the dimension of the kernel $\operatorname{Ker} Q_{2}(A)$;

(ii) the dimension $k_{2}$ of the image $\operatorname{Im} Q_{2}(A)$ is equal to the dimension of the kernel $\operatorname{Ker} Q_{1}(A)$;

(iii) $n=k_{1}+k_{2}$. 
Moreover, there exists a commutative diagram of linear maps:

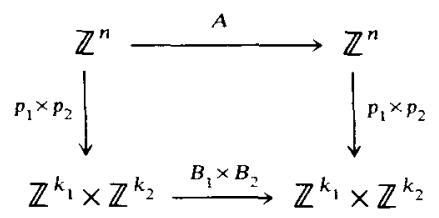

such that:

(iv) the maps $p_{1}$ and $p_{2}$ are surjective;

(v) the minimal polynomial of $B_{1}$ is precisely $Q_{2}$;

(vi) the minimal polynomial of $B_{2}$ is precisely $Q_{1}$;

(vii) the map $p_{1} \times p_{2}$ has a finite coimage; in particular it is an isomorphism over $\mathbb{Q}$.

3. Some results on pseudo-Anosov maps

Definition 3.1. Let $f: M_{g} \rightarrow M_{g}$ be a pseudo-Anosov diffeomorphism of the oriented compact surface of genus $g$. If its invariant foliations are orientable, then we can define the stable foliation by a closed 1 -form $\omega^{s}$ which satisfies $f^{*} \omega^{s}=\lambda \omega^{s}$, with $|\lambda|>1$. We call $\lambda$ the algebraic dilatation coefficient. Of course $|\lambda|$ is the usual dilatation coefficient of $f$.

I learned the following result from Gilbert Levitt [Le1] and [Le2, pp. 208-209]. A different proof is provided by David Fried [Fri].

Lemma 3.2. Suppose that $f: M_{g} \rightarrow M_{g}$ is a pseudo-Anosov diffeomorphism with orientable invariant foliations. Then the algebraic dilatation coefficient is a simple eigenvalue of the action $f_{*}: H^{1}\left(M_{g}, \mathbb{Z}\right) \rightarrow H^{1}\left(M_{g}, \mathbb{Z}\right)$ induced by $f$.

Definition 3.3. if $f$ is a homeomorphism of an orientable manifold, we define $\epsilon(f)$ by:

$$
\epsilon(f)= \begin{cases}+1 & \text { if } f \text { preserves the orientation; } \\ -1 & \text { if } f \text { reverses the orientation. }\end{cases}
$$

Remark 3.4. If $f: \rightarrow M_{g}$ is a homeomorphism of the compact orientable surface of genus $g$, we have $\left(f_{*}\right)^{*} I=\epsilon(f) I$, where $I: H_{1}\left(M_{\mathrm{g}}, \mathbb{Z}\right) \times H_{1}\left(M_{g}, \mathbb{Z}\right) \rightarrow \mathbb{Z}$ is the intersection form. Since $I$ is a symplectic form, if $\mu$ is an eigenvalue of $f_{*}$ then $\epsilon(f) \mu^{-1}$ is also an eigenvalue. In particular, eigenvalues on $H_{1}$ for homeomorphisms of orientable surfaces are in fact algebraic integers.

We have the following lemma:

LemMA 3.5. Let $f: M_{g} \rightarrow M_{g}$ be a pseudo-Anosov diffeomorphism of the compact orientable surface of genus $g$, with orientable invariant foliations, call its algebraic dilatation coefficient $\lambda$. Let $P_{\lambda}(X)$ be the polynomial of $\lambda$ on $\mathbb{Q}$. Consider two cases:

(i) The other eigenvalue $\epsilon(f) \lambda^{-1}$ is conjugate to $\lambda$ over $\mathbb{Q}$, then the degree of $P_{\lambda}$ is $2 g^{\prime}$ for some $g^{\prime}$, with $1 \leq g^{\prime} \leq g$. Moreover, the characteristic and the minimal polynomials of $f_{*}$ admit $P_{\lambda}$ as a simple factor.

(ii) The other eigenvalue $\epsilon(f) \lambda^{-1}$ is not conjugate to $\lambda$ over $\mathbb{Q}$, then $P_{\lambda}$ and $P_{\epsilon(f) \lambda^{-1}}$ are two distinct prime polynomials of the same degree $g^{\prime}$, with $1 \leq g^{\prime} \leq g$. Moreover, 
the characteristic and the minimal polynomials of $f_{*}$ admit $P_{\lambda}$ and $P_{\epsilon(f) \lambda^{-1}}$ as simple factors.

Proof. It is an elementary - though rather long-exercise to prove that, in the first case, the degree of $\vec{\lambda}$ must be even. If we write $P$ for the characteristic polynomial of $f_{*}: H_{1}\left(M_{g}, \mathbb{Z}\right) \rightarrow H_{1}\left(M_{g}, \mathbb{Z}\right)$, then $P_{\lambda}$ appears as a simple factor of $P$. Moreover, by 3.4 , in the second case $P_{\lambda} P_{\epsilon(f) \lambda^{-1}}$ appears as a simple factor. This implies the same facts for the minimal polynomial.

COROLlary 3.6. There exists an automorphism $A: \mathbb{Z}^{2 g^{\prime}} \rightarrow \mathbb{Z}^{2 g^{\prime}}$ for some $g^{\prime}$, with $1 \leq g^{\prime} \leq$ $g$, whose characteristic polynomial is either $P_{\lambda}$ if $\lambda$ is conjugate to $\epsilon(f) \lambda^{-1}$ or $P_{\lambda} P_{\epsilon(f) \lambda^{-1}}$ if not, and there exists a surjective linear map $\alpha: H_{1}\left(M_{g}, \mathbb{Z}\right) \rightarrow \mathbb{Z}^{2 g^{\prime}}$ such that the following diagram is commutative:

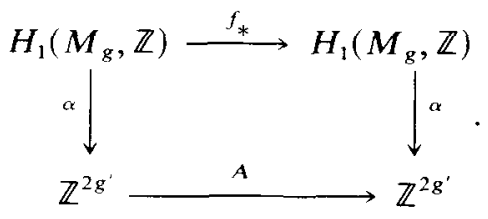

Proof. By 3.5, we can write the minimal polynomial $P_{f_{*}}$ as a product $P_{f_{*}}=Q_{1} Q_{2}$, where $Q_{1}$ and $Q_{2}$ have no common factor and either $Q_{1}=P_{\lambda}$, if $\lambda$ is conjugate to $\epsilon(f) \lambda^{-1}$, or $Q_{1}=P_{\lambda} P_{\epsilon(f) \lambda^{-1}}$, if not. It remains to apply theorem 2.1.

\section{An application of a theorem of John Franks}

A proof of the next theorem can be found in [Fra1];

THEOREM 4.1 (Franks). Let $X$ be a compact space having the homotopy type of a polyhedron. Suppose that $f: X \rightarrow X$ is a homeomorphism of the compact space $X$ and that $A: \mathbb{J}^{n} \rightarrow \mathbb{Z}^{n}$ is an Anosov linear automorphism. If we have a commutative diagram of algebraic maps:

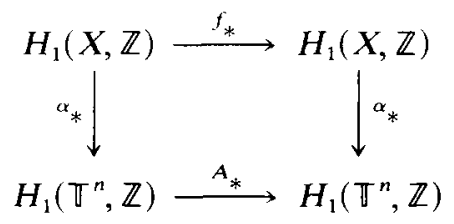

then there exists a continuous map $\alpha: X \rightarrow \mathbb{T}^{n}$, which induces $\alpha_{*}: H_{1}(X, \mathbb{Z}) \rightarrow H_{1}\left(\mathbb{J}^{n}, \mathbb{Z}\right)$ and such that the following diagram:

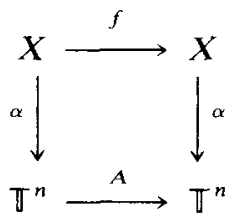

is commutative.

Theorem 4.1 and corollary 3.6 obviously imply the next corollary: 
Corollary 4.2. Suppose that $f: M_{g} \rightarrow M_{g}$ is a pseudo-Anosov diffeomorphism with orientable foliations whose algebraic dilatation coefficient $\lambda$ has no conjugate over $\mathbb{Q}$ which is in $S^{1}$. We can find a commutative diagram

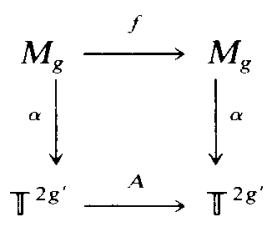

where $A$ is an Anosov linear automorphism whose characteristic polynomial is either $P_{\lambda}$ if $\lambda$ is conjugate to $\epsilon(f) \lambda^{-1}$ or $P_{\lambda} P_{\epsilon(f) \lambda^{-1}}$ if not. Moreover, the induced map $\alpha_{*}: H_{1}\left(M_{g}, \mathbb{Z}\right) \rightarrow H_{1}\left(\mathbb{T}^{2 g^{\prime}}, \mathbb{Z}\right)$ is surjective.

5. The local injectivity argument

The goal of this section is to prove that the map $\alpha$ obtained in 4.2 is almost locally injective.

Lemma 5.1. Suppose that we have a commutative diagram of continuous maps:

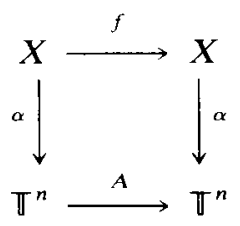

where $A$ is a linear map admitting an eigenvalue $\mu$ and $f: X \rightarrow X$ is a diffeomorphism of the compact connected manifold $X$ which has a closed 1 -form $\omega$ satisfying $f^{*} \omega=$ $\mu \omega$ - where the equality is on the level of forms and not only on cohomology. Call $\Omega$ the linear 1-form on the torus which satisfies $A^{*} \Omega=\mu \Omega$. Suppose moreover, that on the cohomology level we have $\alpha^{*}[\Omega]=[\omega]$. Then if $|\mu|>1$, we have the equality: $\alpha^{*} \tilde{\Omega}=\tilde{\omega}$ in $\mathscr{C}^{1}(X)$.

Proof. We can write:

$$
\alpha^{*} \tilde{\Omega}=\tilde{\omega}+d \varphi
$$

where $\varphi \in \mathscr{C}(X)$. If we apply $f$ to this equation and use the fact that $\alpha f=A \alpha$ we obtain:

$$
\mu \alpha^{*} \tilde{\Omega}=\mu \tilde{\omega}+d(\varphi f)
$$

Comparing equations (1) and (2) gives:

$$
d(\varphi f-\mu \varphi)=0 \text {. }
$$

Since $X$ is connected, we can find $k \in \mathbb{C}$ such that:

$$
\varphi f=\varphi+k \text {. }
$$

This implies that the compact set $\operatorname{Im} \varphi$ is invariant under the linear hyperbolic map $\mathbb{C} \rightarrow \mathbb{C}, z \mapsto \mu z+k$. It follows that this image is reduced to the fixed point of that linear map. In particular, the map $\varphi$ is constant. This finishes the proof by equation (1). 
THEOREM 5.2. Suppose that $f: M_{g} \rightarrow M_{g}$ is a pseudo-Anosov diffeomorphism with orientable foliations whose algebraic dilatation coefficient $\lambda$ has no conjugate over $\mathbb{Q}$ which is in $S^{1}$. Consider the commutative diagram given by 4.2:

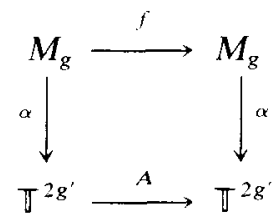

where $A$ is an Anosov linear automorphism whose characteristic polynomial is either $P_{\lambda}$ if $\lambda$ is conjugate to $\epsilon(f) \lambda^{-1}$ or $P_{\lambda} P_{\epsilon(f) \lambda^{-1}}$ if not.

The map $\alpha$ is injective on each small enough rectangle of the local product structure given by the invariant foliations of $f$.

Proof. Choose $\epsilon>0$ such that a ball of radius less than $\epsilon$ in $\mathbb{T}^{2 g^{\prime}}$ is contractible. Choose $\delta>0$ such that each $\delta$-ball in $M_{\mathrm{g}}$ is contractible and has an image under $\alpha$ contained in an $\epsilon$-ball of $\mathbb{T}^{2 g^{\prime}}$. Given $x$ in $M_{g}$ (resp. $\mathbb{T}^{2 g^{\prime}}$ ), there exists a well defined map $\omega_{x}: B(x, \delta) \rightarrow \mathbb{R}^{2}$ (resp. $\Omega_{x}: B(x, \epsilon) \rightarrow \mathbb{R}^{2}$ ) such that the value of the first and second coordinates at a point $y$ are obtained by integrating 1 -forms $\omega^{\text {s }}, \omega^{\prime \prime}$ defining the stable and unstable foliations (resp. linear forms $\Omega^{s}, \Omega^{u}$ with $\alpha^{*}\left[\Omega^{s}\right]=$ $\left[\omega^{s}\right]$ and $\alpha^{*}\left[\Omega^{u}\right]=\left[\omega^{u}\right]$ in cohomology) on any path joining $x$ to $y$ and contained in $B(x, \delta)$ (resp. $B(x, \epsilon)$ ). It is easy to see that $\omega_{x}$ is injective on each rectangle defined by the invariant foliations which is contained in $B(x, \delta)$. But lemma 5.1 shows that $\omega_{x}=\Omega_{\alpha(x)} \alpha$.

6. An upper bound for the capacity of a compact set invariant under a linear automorphism of a torus

The goal of this section is to prove a general upper estimate on the Hausdorff dimension of a compact set invariant by a linear automorphism of a torus. The proof is a simple application of the Douady-Oesterlé trick [DO] which allows to use parallelotopes instead of cubes. This is also an easy instance of the work of Ledrappier and Young [LY] though it is not formally a consequence of their result.

In fact, we will obtain a bound for the upper capacity.

We recall the definition of upper capacity - also called capacity. If $X$ is a metric space, we denote by $N(X, \epsilon)$ the minimum number of $\epsilon$-balls covering $X$. The (upper) capacity $C(X)$ of $X$ is:

$$
C(X)=\limsup _{\epsilon \rightarrow 0} \frac{N(X, \epsilon)}{-\log \epsilon} .
$$

In the same way, the lower capacity $C(X)$ of $X$ is:

$$
C(X)=\liminf _{\epsilon \rightarrow 0} \frac{N(X, \epsilon)}{-\log \epsilon} .
$$

If we denote by $\operatorname{HD}(X)$ the Hausdorff dimension of $X$, we have the inequalities:

$$
\operatorname{HD}(X) \leq C(X) \leq C(X) .
$$


Remark that the quantities $\operatorname{HD}(X), C(X)$ and $C(X)$ remain the same if we replace the metric on $X$ by one which is Lipschitz equivalent.

The next lemma is of course well-known; we provide its simple proof.

Lemma 6.1. Suppose that $E$ is a normed space of finite dimension $d$. There exists a constant $k$ such that for every $r, R \in \mathbb{R}$, with $r \leq R$, a ball of radius $R$ can be covered by at most $k(R / r)^{d}$ balls of radius $r$.

Proof. We denote by $\|\cdot\|$ the norm on $E$. We endow $\mathbb{R}^{d}$ with the max norm, which we denote by $|\cdot|$. We identify $\mathbb{R}^{d}$ with $E$. There exists $C \geq 1$ such that:

$$
\forall x \in E, \quad C^{-1}|x| \leq\|x\| \leq C|x| .
$$

We call $B(x, s)$ (resp. $B^{\prime}(x, s)$ ) the ball of center $x$ and radius $s$ in the norm $\|\cdot\|$ (resp.|.|). We have:

$$
\forall x \in E, \forall s \in \mathbb{R}_{+}, \quad B^{\prime}(x, s) \subset B(x, C s) \quad \text { and } \quad B(x, s) \subset B^{\prime}(x, C s) .
$$

To prove the lemma, we may assume that $R=1$ and $r \leq 1$. The unit ball $B(0,1)$ in the norm $\|\cdot\|$ is contained in the ball $B^{\prime}(0, C)$. Since this last norm $|\cdot|$ is the $\max$ norm, it is possible to cover $B^{\prime}(0, C)$ by at most $\left(\left[C /\left(C^{-1} r\right)\right]+1\right)^{d} \leq 2^{d}\left(C^{2} / r\right)^{d}$ balls of radius $C^{-1} r$ in the norm $|\cdot|$. By the inequality on the norms, a ball of radius $C^{-1} r$ in the norm $|\cdot|$ is contained in a ball of radius $r$ in the norm $\|\cdot\|$. It follows that we can take $k=2^{d} C^{2 d}$.

The following fact is an easy observation which is very useful.

LemMA 6.2. Let $\varphi:] 0, \infty[\rightarrow \mathbb{R}$ be a decreasing function. If $\theta \in] 0,1\left[\right.$ and $\epsilon_{0}>0$, we have:

$$
\begin{gathered}
\limsup _{\epsilon \rightarrow 0} \frac{\log \varphi(\epsilon)}{-\log \epsilon}=\limsup _{n \rightarrow \infty} \frac{\log \varphi\left(\theta^{n} \epsilon_{0}\right)}{-\log \theta^{n} \epsilon_{0}} \\
\liminf _{\epsilon \rightarrow 0} \frac{\log \varphi(\epsilon)}{-\log \epsilon}=\liminf _{n \rightarrow \infty} \frac{\log \varphi\left(\theta^{n} \epsilon_{0}\right)}{-\log \theta^{n} \epsilon_{0}} .
\end{gathered}
$$

Proof. To simplify notations, we will suppose that $\epsilon_{0}=1$. Define:

$$
n(\epsilon)=\left[\frac{\log \epsilon}{\log \theta}\right] \text {. }
$$

Since $\theta \in] 0,1[$, we have:

$$
\lim _{\epsilon \rightarrow 0} n(\epsilon)=\infty \quad \text { and } \quad \lim _{\epsilon \rightarrow 0} \frac{\log \epsilon}{\log \theta^{n(\epsilon)}}=1 .
$$

Since $\varphi$ is decreasing, we have by the definition of $n(\epsilon)$ :

$$
\varphi\left(\theta^{n(\epsilon)}\right) \leq \varphi(\epsilon) \leq \varphi\left(\theta^{n(\epsilon)+1}\right)
$$

For $\epsilon<1$, this gives:

$$
\frac{\log \varphi\left(\theta^{n(\epsilon)}\right)}{-\log \epsilon} \leq \frac{\log \varphi(\epsilon)}{-\log \epsilon} \leq \frac{\log \varphi\left(\theta^{n(\epsilon)+1}\right)}{-\log \epsilon} .
$$

The result follows from (1) and (3). 
THEOREM 6.3. Suppose that $A: \mathbb{T}^{n} \rightarrow \mathbb{T}^{n}$ is a linear automorphism of a torus. Let us denote by $\lambda_{1}, \ldots, \lambda_{n}$ its eigenvalues counted with multiplicities. For each $\lambda_{j}$, define $\tau_{j}$ by:

$$
\tau_{j}=\left\{\begin{aligned}
\log \left|\lambda_{j}\right| & \text { if }\left|\lambda_{j}\right| \leq 1 \\
-\log \left|\lambda_{j}\right| & \text { if }\left|\lambda_{j}\right| \geq 1 .
\end{aligned}\right.
$$

By renumbering $\tau_{1}, \ldots, \tau_{n}$, we will suppose that $\tau_{1} \geq \tau_{2} \geq \cdots \geq \tau_{n}$. Define $\tau:[0, n] \rightarrow \mathbb{R}$ by $\tau(s)=\tau_{1}+\cdots+\tau_{[s]}+(s-[s]) \tau_{[s]+1}$. This function is negative and decreasing.

Let $K \subset \mathbb{T}^{n}$ be a compact set invariant under $A$, call $\lambda$, the topological entropy of A|K. Define:

$$
D=\sup \{s \in[0, n] \mid 2 \lambda=-\tau(s)\} .
$$

We have the inequality:

$$
C(K) \leq D \text {. }
$$

Proof: Decompose $\mathbb{R}^{n}$ as a direct sum $E_{1} \oplus \cdots \oplus E_{k}$, where $A$ has all its eigenvalues on $E_{j}$ contained in the circle of radius $r_{j}$. Define in the same way as above:

$$
t_{j}=\left\{\begin{aligned}
\log r_{j} & \text { if } r_{j} \leq 1 \\
-\log r_{j} & \text { if } r_{j} \geq 1
\end{aligned}\right.
$$

Without loss of generality, we can assume that $t_{j+1} \leq t_{j}$. If we define $t:[0, n] \rightarrow \mathbb{R}$ by $t(s)=\sum_{1 \leq i<j} \operatorname{dim}\left(E_{i}\right) t_{i}+(s-[s]) t_{j}$ if $\sum_{1 \leq i<j} \operatorname{dim}\left(E_{i}\right) \leq s \leq \sum_{1 \leq i \leq j} \operatorname{dim}\left(E_{i}\right)$. It is easy to see that $t=\tau$.

Fix $\epsilon>0$. For future purposes, we will suppose that $t_{i}+\epsilon<0$ for each $i$ such that $t_{i}<0$. Fix $j \in\{1, \ldots, k\}$ such that $t_{j}<0$. It is possible to choose a norm $\|\cdot\|$ on $\mathbb{R}^{n}$, which is the maximum of norms on the $E_{i}$ and such that $\inf \left(\log \left\|A\left|E_{i}\|, \log \| A^{-1}\right| E_{i}\right\|\right) \leq t_{i}+\epsilon$. Call $\mathscr{B}_{\delta}$ the covering of $\pi^{n}$ by $\delta$-balls in the norm $\|\cdot\|$. Call $\mathcal{N}(n, \epsilon)$ the minimum number of elements in $\mathscr{B}_{\delta}(n)=\bigvee_{i=0}^{n} A^{-i}\left(\mathscr{B}_{\delta}\right)$ covering $K$. By the definition of entropy we have:

$$
\lim _{\delta \rightarrow 0} \limsup _{n \rightarrow \infty} \frac{\log \mathcal{N}(n, \delta)}{n}=\lambda \text {. }
$$

Remark that each element in $A^{n}\left(\mathscr{B}_{\delta}(2 n)\right)$ is contained in a set which is the product of balls in each of the $E_{i}$, where the ball in $E_{i}$ has radius $\delta \exp n\left(t_{i}+\epsilon\right)$. From lemma 6.1, it follows that such a set is contained in at most $C_{j} \exp \left[\sum_{i=1}^{j-1} n \operatorname{dim}\left(E_{i}\right) \times\right.$ $\left.\left(t_{i}-t_{j}\right)\right]$ balls of radius $\delta \exp n\left(t_{j}+\epsilon\right)$, where $C_{j}$ is a constant independent of $n$ and $\delta$. We can find $\delta_{0}$ such that:

$$
\forall \delta \leq \delta_{0}, \limsup _{n \rightarrow \infty} \frac{\log \mathcal{N}(n, \delta)}{n} \leq \lambda+\frac{\epsilon}{2} .
$$

In particular, we can choose $n\left(\delta_{0}\right)$ such that:

$$
\forall n \geq n\left(\delta_{0}\right), \quad \frac{\log \mathcal{N}\left(n, \delta_{0}\right)}{n} \leq \lambda+\epsilon .
$$

From the estimates above, we obtain that:

$$
\begin{aligned}
\forall n & \geq n\left(\delta_{0}\right), \quad \frac{\log N\left(K, \delta_{0} \exp n\left(t_{j}+\epsilon\right)\right)}{-n\left(t_{j}+\epsilon\right)} \\
& \leq \frac{2(\lambda+\epsilon)}{-\left(t_{j}+\epsilon\right)}+\frac{\log C_{j}}{-n\left(t_{j}+\epsilon\right)}+\frac{\sum_{i=1}^{j-1} \operatorname{dim}\left(E_{i}\right)\left(t_{i}-t_{j}\right)}{-\left(t_{j}+\epsilon\right)} .
\end{aligned}
$$


By lemma 6.2 , this implies:

$$
C(K) \leq \frac{2(\lambda+\epsilon)+\sum_{i=1}^{j-1} \operatorname{dim}\left(E_{i}\right)\left(t_{i}-t_{j}\right)}{-\left(t_{j}+\epsilon\right)} .
$$

Of course, our estimates used a norm on $\mathbb{R}^{n}$ which depended on $\epsilon>0$. Since the left hand side of the above inequality does not depend on the norm, we obtain:

$$
C(K) \leq \frac{2 \lambda+\sum_{i=1}^{j-1} \operatorname{dim}\left(E_{i}\right)\left(t_{i}-t_{j}\right)}{-t_{j}} .
$$

The above inequality is valid for all $j \in\{1, \ldots, k\}$ such that $t_{j}<0$. We leave it as an exercise to show that the minimum over these $j$ of the left hand side of the last inequality is precisely $D$.

\section{A lower bound for the capacity}

From the work of Urbański [Ur, Theorem 1], it is possible to obtain a general lower bound for the capacity of $\alpha\left(M_{\mathrm{g}}\right)$ - where $\alpha$ is associated by 4.2 to a pseudo-Anosov map $f: M_{g} \rightarrow M_{g}$. This was brought to my attention by Feliks Przytycki during our stay in spring 1986 at the University of Warwick for the 'Special Year in Smooth Ergodic Theory'. In fact, the argument of Urbański can be used to prove a slightly better result than what can be formally obtained from [Ur, Theorem 1].

THEOREM 7.1. Let $f: M_{g} \rightarrow M_{g}$ be a pseudo-Anosov map, with orientable stable and unstable foliations and whose algebraic dilatation $\lambda$ coefficient has no conjugate on the unit circle. Suppose that $\lambda$ is not a quadratic integer. Call $P_{\lambda}$ the polynomial of $\lambda$ over Q. Call $\mu_{1}>\cdots>\mu_{h}$ the elements of the set $\left\{|\rho| \mid P_{\lambda}(\rho) P_{\epsilon(f) \lambda^{-1}}(\rho)=0\right.$, with $\left.|\rho|>1\right\}$. Since $|\lambda|$ appears as the biggest eigenvalue of an irreducible positive matrix, we have: $\mu_{1}=|\lambda|$. Moreover, since $\lambda$ is not quadratic, we have: $h \geq 2$. If $\alpha: M_{g} \rightarrow \mathbb{J}^{2 g}$ is the map associated to f by 4.2 , we have:

$$
C\left(\alpha\left(M_{g}\right)\right) \geq 2\left(1+\sum_{i=1}^{h-1}\left(1+-\frac{\log \mu_{i+1}}{\log \mu_{i}}\right)\right) .
$$

Because $h \geq 2$, this implies that $\underline{C}\left(\alpha\left(M_{g}\right)\right)>2$.

We will need some lemmas:

LEMMA 7.2. Suppose that for $j=1, \ldots, k$, we have $\varphi_{j}:[0,1] \rightarrow E_{j}$, a continuous function from the interval $[0,1]$ to the finite dimensional normed space $E_{j}$. Suppose that these continuous functions satisfy the following property:

$\exists C>0, \epsilon_{1}, \ldots, \epsilon_{k-1}, 0<\epsilon_{j}<1$, such that:

$$
\forall[a, b] \subset[0,1], \quad \max _{t \in[a, b]}\left\|\varphi_{j+1}(t)-\varphi_{j+1}(a)\right\| \geq C\left\|\varphi_{j}(b)-\varphi_{j}(a)\right\| \epsilon_{j} .
$$

Then if $\varphi_{1}$ is not constant, the multigraph $G=\left\{\left(\varphi_{1}(t), \ldots, \varphi_{k}(t)\right) \mid t \in[0,1]\right\}$ has a lower bound for its lower capacity which is given by:

$$
C(G) \geq 1+\sum_{i=1}^{k-1}\left(1-\epsilon_{i}\right)
$$

Proof. For each $i=1, \ldots, k$, we define $E^{i}=\bigoplus_{j=1}^{i} E_{j}$ and we endow $E^{i}$ with the norm $\left\|\left(x_{1}, \ldots, x_{i}\right)\right\|=\max _{j=1}^{i}\left\|x_{j}\right\|$. 
For each integer $n \geq 1$ and each $j=1, \ldots, k$, we are going to define, by induction on $j=1, \ldots, k$, a finite family $\mathscr{F}_{n}^{j}$ of balls in $E^{j}$ such that:

(i) the balls in $\mathscr{F}_{n}^{j}$ have radius $2^{-n}$;

(ii) the balls in $\mathscr{F}_{n}^{j}$ have disjoint interiors;

(iii) given a ball $B$ in $\mathscr{F}_{n}^{j}$, there exists a subinterval $[a, b] \subset[0,1]$ such that:

$$
\left.\left\{\left(\varphi_{1}(t), \ldots, \varphi_{j}(t)\right) \mid t \in[a, b]\right)\right\} \subset B \text { and }\left\|\varphi_{j}(b)-\varphi_{j}(a)\right\|=2^{-n}
$$

(iv) the number $\# \mathscr{F}_{n}^{j}$ of balls in $\mathscr{F}_{n}^{j}$ satisfies:

$$
\begin{aligned}
& \# \mathscr{F}_{n}^{1} \geq\left[\left\|\varphi_{1}(1)-\varphi_{1}(0)\right\| 2^{n-1}\right] \\
& \# \mathscr{F}_{n}^{j} \geq \mathscr{F}_{n}^{j-1}\left[C 2^{-n \epsilon_{j-1} 2^{n-1}}\right],
\end{aligned}
$$

where $[r]$ is the integer part of the real number $r$.

Suppose that we have already obtained $\mathscr{F}_{n}^{j}$. If $B$ is a ball in $\mathscr{F}_{n}^{j}$, consider the interval $[a, b]$ associated to $B$ by (iii), applying the hypothesis of the lemma, we obtain:

$$
\left\|\varphi_{j+1}(b)-\varphi_{j+1}(a)\right\| \geq C 2^{-n \epsilon_{i}} .
$$

It follows that we can construct $\left[C 2^{-n \epsilon_{i}} 2^{n-1}\right]$ balls in $E^{j+1}$ of radius $2^{-n}$ which have disjoint interiors, satisfy the analogous of condition (iii) with $j$ replaced by $j+1$ and whose projection on $E^{j}$ is contained in $B$. If we apply this to all balls $B$ in $\mathscr{F}_{n}^{j}$, we obtain the family $\mathscr{F}_{n}^{j+1}$.

It follows from (iv) that there exists some $A>0$ such that:

$$
\forall n \geq 1, \quad \# \mathscr{F}_{n}^{k} \geq\left(A 2^{n}-1\right) \prod_{i=1}^{k-1}\left(A 2^{n\left(1-\epsilon_{i}\right)}-1\right) .
$$

Since $E^{k}$ is finite dimensional, there exists, by volume consideration, a number $K$ such that for any $r>0$, at most $K$ disjoint open balls of radius $r$ can be contained in the same ball of radius $3 r$. It follows that at most $K$ disjoint open balls of radius $r$ can intersect the same ball of radius $r$. This last fact, joined to inequality (1), shows that any cover of the multigraph $G$ by balls of radius $2^{-n}$ must contain at least $K^{-1}\left(A 2^{n}-1\right) \prod_{i=1}^{k-1}\left(A 2^{n\left(1-\varepsilon_{i}\right)}-1\right)$ elements. Hence, by 6.2 , we get:

$$
C(G) \geq 1+\sum_{i=1}^{k-1}\left(1-\epsilon_{i}\right) .
$$

In the following, we will consider a pseudo-Anosov map $f: M_{g} \rightarrow M_{g}$ satisfying the hypothesis of 7.1. We call $\alpha: M_{g} \rightarrow \mathbb{J}^{2 g^{\prime}}$ and $A: \mathbb{J}^{2 g^{\prime}} \rightarrow \mathbb{T}^{2 g^{\prime}}$ the two maps associated to $f$ by 4.2 . We will also call $A$ the linear lift of the map $A: \mathbb{T}^{2 g^{\prime}} \rightarrow \mathbb{T}^{2 g^{\prime}}$ to $\mathbb{R}^{2 g^{\prime}}$. Although with some more routine work we can dispense with the fact that the characteristic polynomial of $A$ has no multiple roots, we use that fact to write:

$$
\mathbb{R}^{2 g^{\prime}}=\bigoplus_{i=1}^{2 k} E_{i}
$$

with $A\left(E_{i}\right)=E_{i}$ and

$$
\|A(x)\|=\left\{\begin{aligned}
\mu_{i}\|x\|, & \text { if } x \in E_{i}, \text { for } i=1, \ldots, k \\
\mu_{i-k}^{-1}\|x\|, & \text { if } x \in E_{i}, \text { for } i=k+1, \ldots, 2 k
\end{aligned}\right.
$$

We will consider the collection $\mathscr{F}$ of continuous functions from $\mathbb{R}$ to $\mathbb{R}^{2 g^{\prime}}$ defined in the following way: a map is in $\mathscr{F}$ if it is the composition of an isometry of $\mathbb{R}$ 
into a union of unstable leaves and singularities of the unstable foliation lifted to the universal cover $\tilde{M}_{g}$ of $M_{g}$ with the lift $\tilde{\alpha}: \tilde{M}_{g} \rightarrow \mathbb{R}^{2 g^{\prime}}$ of $\alpha$ to the universal covers.

LEMMA 7.3. The family $\mathscr{F}$ satifies the following properties:

(i) if $\varphi$ is in $\mathscr{F}$, then $t \mapsto \varphi(t+r)$ is in $\mathscr{F}$ for any $r \in \mathbb{R}$;

(ii) if $\varphi$ is in $\mathscr{F}$, then $t \mapsto A^{-n} \varphi\left(|\lambda|^{n} t\right)$ is in $\mathscr{F}$ for any $n \in \mathbb{Z}$;

(iii) if $\varphi$ is in $\mathscr{F}$, write $\varphi=\left(\varphi_{1}, \ldots, \varphi_{2 k}\right)$ with $\varphi_{i}:[0,1] \rightarrow E_{i}$, then for $i=k+1, \ldots, 2 k$, we have $\varphi_{i}=0$.

Proof. The first property is evident from the definition. The second one follows from the fact that $\alpha$ is a semi-conjugacy. The third one follows from the fact that $\alpha$ takes leaves of the unstable foliation of $f$ into leaves of the unstable foliation of A.

LEMMA 7.4. If $\left(\varphi_{n}\right)_{n \in \mathbb{N}}$ is a sequence in $\mathscr{F}$, there exists a sequence $\left(v_{k}\right)_{k \in \mathbb{N}}$ of vectors $v_{k} \in \mathbb{Z}^{2 g^{\prime}}$ and a subsequence $\varphi_{n_{k}}$ such that $\varphi_{n_{k}}+v_{k} \in \mathscr{F}$ and the sequence $\left(\varphi_{n_{k}}+v_{k}\right)_{k \in \mathbb{N}}$ converges to a function $\varphi \in \mathscr{F}$.

Proof. This follows easily from the fact that the unstable foliation is continuous and from the compactness of $M_{g}$.

LEMMA 7.5. If $\varphi \in \mathscr{F}$ is written as $\varphi=\left(\varphi_{1}, \ldots, \varphi_{2 k}\right)$ with $\varphi_{i}:[0,1] \rightarrow E_{i}$, then for $i=1, \ldots, k$, the map $\varphi_{i}$ cannot be constant on an interval which is not reduced to a point.

Proof. If some $\varphi_{i}$ were constant on some non trivial interval, then we could find a non trivial unstable segment $S$ in $M_{\mathrm{g}}$ whose image under $\alpha$ would be contained in a codimension $\geq 1$ 'linear' subspace $P$ of some unstable leaf of $A: \mathbb{T}^{2 g^{\prime}} \rightarrow \mathbb{T}^{2 g^{\prime}}$. Since the stable leaves of $f$ in $M_{\mathrm{g}}$ are dense and since $\alpha$ takes stable leaves of $f$ to stable leaves of $A$, it follows that $\alpha\left(M_{g}\right)$ is contained in the union of the stable leaves of $A$ that intersect $P$. This last union is a codimension $\geq 1$ 'linear' subspace in $\mathbb{T}^{2 g^{\prime}}$. This is a contradiction since the inclusion of $P \subset \mathbb{J}^{2} g^{\prime}$ is not surjective on the first homology group but $\alpha$ is.

LEMMA 7.6. (A priori inequalities). There exists a number $C>0$ such that if $\varphi \in \mathscr{F}$ is written as $\varphi=\left(\varphi_{1}, \ldots, \varphi_{2 k}\right)$ with $\varphi_{i}:[0,1] \rightarrow E_{i}$, then we have:

$$
\forall j=1, \ldots, k-1, \forall[a, b] \subset \mathbb{R}, \quad \max _{t \in[a, b]}\left\|\varphi_{j+1}(t)-\varphi_{j+1}(a)\right\| \geq C\left\|\varphi_{j}(b)-\varphi_{j}(a)\right\|^{\epsilon_{j}}
$$

where $\epsilon_{j}=\left(\log \mu_{j+1}\right) /\left(\log \mu_{j}\right)$, for $j=1, \ldots, k-1$.

Proof. Suppose that the conclusion is false. Using the properties of $\mathscr{F}$ given in lemma 7.3, and selecting some subsequence, if necessary, we can find a $j \in$ $\{1, \ldots, k-1\}$ and sequences $\varphi^{n} \in \mathscr{F}$ and $t_{n} \geq 0$ such that:

$$
\max _{0 \rightarrow 1=t_{n}}\left\|\varphi_{j+1}^{n}(t)-\varphi_{j+1}^{n}(0)\right\|<\frac{1}{n}\left\|\varphi_{j}^{n}\left(t_{n}\right)-\varphi_{j}^{n}(0)\right\|^{\epsilon_{i}} .
$$

Above and in the following: if $\psi: \mathbb{R} \rightarrow \mathbb{R}^{2 g^{\prime}}$, then $\psi_{l}$ is the $l$-th component of $\psi$ with respect to the decomposition $\mathbb{R}^{2 g^{\prime}}=\bigoplus_{i=1}^{2 k} E_{i}$.

Remark that (1) implies that in fact $t_{n}>0$. In particular, we can find for each $n$ an integer $m_{n} \in \mathbb{Z}$ such that $|\lambda|>|\lambda|^{m_{n}} t_{n} \geq 1$. If we define $t_{n}^{\prime}=|\lambda|^{m_{n}} t_{n}$ and $\varphi^{\prime n}(t)=$ $A^{m_{n}} \varphi^{n}\left(|\lambda|^{m_{n}} t\right)$, we have $\varphi^{\prime n} \in \mathscr{F}$ and $t_{n}^{\prime} \in[1,|\lambda|]$. From the definition of the 
decomposition $\mathbb{R}^{2 g^{\prime}}=\bigoplus_{i=1}^{2 k} E_{i}$, we have:

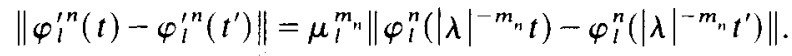

Now it is clear that $(1),(2)$ and the definition of $\epsilon_{j}$ imply the following:

$$
\max _{0 \leq t \leq 1}\left\|\varphi_{j+1}^{\prime n}(t)-\varphi_{j+1}^{\prime n}(0)\right\|<\frac{1}{n} \max _{0 \leq t \leq \lambda}\left\|\varphi_{j}^{\prime n}(t)-\varphi_{j}^{\prime n}(0)\right\|^{\epsilon_{j}} .
$$

By 7.4, we can find vectors $v_{n} \in \mathbb{Z}^{2 g^{\prime}}$ and a function $\varphi \in \mathscr{F}$ such that there is a subsequence of $\varphi^{\prime n}+v_{n}$ whose uniform limit on each compact subset of $\mathbb{R}$ is $\varphi$. But (3) in the limit shows that $\varphi_{j+1}$ is constant on the interval $[0,1]$. This contradicts 7.5 .

Proof of 7.1. It follows from what was done above that if $S$ (resp. $U$ ) is a stable (resp. unstable) small non trivial segment in $M_{g}$ then $C(\alpha(S)) \geq 1+\sum_{i=1}^{k-1}\left(1-\epsilon_{i}\right)$ (resp. $C(\alpha(U)) \geq 1+\sum_{i=1}^{k-1}\left(1-\epsilon_{i}\right)$ ). In fact $\alpha(S)$ (resp. $\left.\alpha(U)\right)$ is contained in a stable (resp. unstable) leaf of $A$ and $\alpha$ respects the local product structures. Moreover, it is a general fact that for subsets of euclidean spaces the lower capacity of a cartesian product is $\geq$ the sum of the lower capacity of its factors. This finishes the proof of 7.1

\section{Pseudo-Anosov diffeomorphisms with Pisot dilatation coefficient}

We consider in the following a pseudo-Anosov diffeomorphism $f: M_{g} \rightarrow M_{g}$ with orientable invariant foliations and an algebraic dilatation coefficient $\lambda$ which is a Pisot number, i.e. all conjugates on $\mathbb{Q}$ have absolute value $<1-$ see the examples given by Arnoux and Yoccoz [AY]. We will suppose that the degree of $\lambda$ on $\mathbb{Q}$ is $g^{\prime}$, this implies that the genus $g$ of $M_{g}$ is $\geq g^{\prime}$. We can apply 4.2 to obtain a commutative diagram:

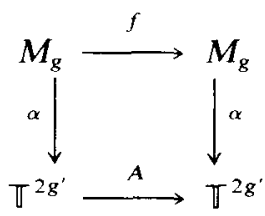

where $A$ is an Anosov linear automorphism whose characteristic polynomial is $P_{\lambda} P_{\epsilon(f) \lambda^{-1}}$.

Using 2.1, we can obtain another commutative diagram:

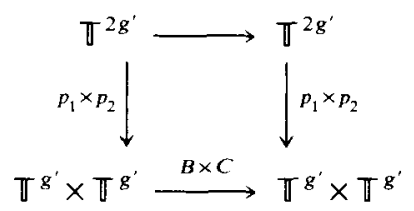

where $B$ (resp. $C$ ) is an Anosov linear automorphism whose characteristic polynomial is $P_{\lambda}$ (resp. $P_{\epsilon(f) \lambda^{-1}}$ ). Of course the map $p_{1} \times p_{2}$ is a covering.

LEMMA 8.1. The maps $p_{1} \alpha, p_{2} \alpha: M_{g} \rightarrow \mathbb{T}^{g^{\prime}}$ are surjective.

Proof. This follows from [Hi, Theorem 5, p. 130]. Here is a slightly different proof. Consider, for example, the case of $\beta=p_{1} \alpha$. A half unstable leaf through a point $x$ 
in $M_{g}$ goes under $\beta$ on a connected subset of the unstable leaf of $\beta(x)$ in $\mathbb{T}^{g^{\prime}}$ which is a line - recall that all conjugates of $\lambda$ have modulus $<1$. Consider the case where the half leaf through $x$ is infinite. If its image is contained in a bounded interval of the unstable leaf of $\beta(x)$ in $\mathbb{T}^{g^{\prime}}$, then the whole image of $M_{g}$ under $\beta$ would be contained in that interval, because we know that the closure in $M_{\mathrm{g}}$ of an infinite half leaf is equal to $M_{\mathrm{g}}$. This is impossible because the map induced by $\beta$ on the first rational homology group is surjective. This implies that the image of a half unstable leaf through $x$ which is infinite contains a half infinite unstable leaf through $\beta(x)$. Such a half unstable leaf in $\mathbb{T}^{g^{\prime}}$ is dense.

LeMMA 8.2. Any segment contained in a stable leaf of $M_{g}$ which is not reduced to a point has an image under $\beta=p_{1} \alpha$ which contains an open subset - in the topology of the leaf - of the corresponding $(g-1)$-dimensional stable leaf in $\mathbb{T}^{g^{\prime}}$.

Proof. Suppose that some compact stable segment $s \subset M_{g}$ which is not reduced to a point has an image $\beta(s)$ with empty interior in the corresponding stable leaf in $\mathbb{T}^{g^{\prime}}$. It is easy to show that, in this case, the union $U$ of the unstable leaves - for $\boldsymbol{A}$ - through some point of $\boldsymbol{\beta}(s)$ is a countable family of compact subsets of $\mathbb{T}^{g^{\prime}}$ with empty interiors. Of course, if we call $V$ the union of the unstable leaves - for $f$-through some point of $s$ this a set which is equal to $M_{g}$ minus a finite number of points - namely the singularities of the invariant foliations. The image $\beta(V)$ is contained in $U$. It follows clearly that the compact set $\beta\left(M_{g}\right)$ is contained in a countable family of compact subsets of $\mathbb{T}^{g^{\prime}}$ with empty interiors. This contradicts Baire's theorem since $\beta\left(M_{g}\right)=\pi^{g^{\prime}}$.

LeMma 8.3. Consider a point $x$ in $M_{g}$ with $z=\alpha(x)$. Call $\pi_{z}$ a local linear projection of a neighborhood of $z$ in $\mathbb{T}^{2 g^{\prime}}$ on the subspace $W_{z}$ through $z$ which is generated by the eigenspaces of $A$ which corresponds to the eigenvalues different from $\lambda$ and $\lambda^{-1}$. Remark that the dimension of $W_{z}$ is $2 g^{\prime}-2$. The image under $\pi_{z} \alpha$ of each small neighborhood of $x$ in $M_{g}$ contains an open subset of $W_{z}$.

Proof. This follows from 8.2. In fact, the map $\pi_{z} \alpha$ can be identified locally with the cartesian product of the two maps $p_{1} \beta$ and $p_{2} \beta$ restricted respectively to a small stable and unstable segments of $f$ containing $x$.

THEOREM 8.4. For each subset $X$ of $M_{g}$ with non-empty interior, we have:

$$
\operatorname{HD}(\alpha(X))=C(\alpha(X))=C(\alpha(X))=2 g^{\prime}-2 .
$$

Proof. From 8.3, near each point of $\alpha\left(M_{g}\right)$, there is a local projection of $\mathbb{T}^{2 g^{\prime}}$ on a codimension 2 'linear' subspace which take any non empty small open subset in $M_{\mathrm{g}}$ to a set with non empty interior in the codimension 2 subspace. This shows that:

$$
\mathrm{HD}(\alpha(X)) \geq 2 g^{\prime}-2 \text {. }
$$

If we call $\mu_{1}, \ldots, \mu_{g^{\prime}-1}$, the conjugate of $\lambda$ on $\mathbb{Q}$, since $\lambda$ is a Piset number we have:

$$
\lambda^{-1}= \pm \mu_{1} \cdots \mu_{g^{\prime}-1}, \quad\left|\mu_{1}\right|, \ldots,\left|\mu_{g^{\prime}-1}\right|<1,
$$

which implies $\left|\lambda^{-1}\right|<\left|\mu_{i}\right|$, for $i=1, \ldots, g^{\prime}-1$. If we suppose that $\mu_{1_{1}}, \ldots, \mu_{1_{g^{\prime}-1}}$ are ordered in such a way that: $\left|\mu_{1}\right| \geq \cdots \geq\left|\mu_{g^{\prime}-1}\right|$, then the numbers $\tau_{1}, \ldots, \tau_{2 g^{\prime}}$ of 
theorem 6.3 satisfy:

$$
\tau_{2 i-1}=\tau_{2 i}=\log \left|\mu_{i}\right|, \text { for } 1, \ldots, g^{\prime}-1
$$

and

$$
\tau_{2 g^{\prime}-1}=\tau_{2 g^{\prime}}=\log \left|\lambda^{-1}\right| \text {. }
$$

This implies that $\tau\left(2 g^{\prime}-2\right)=2 \sum_{i=1}^{g^{\prime}-1} \log \left|\mu_{i}\right|=2 \log \prod_{i=1}^{g^{\prime}-1} \log \left|\mu_{i}\right|=-2 \log |\lambda|$. Since no $\tau_{i}$ is zero, the function $\tau(s)$ is strictly decreasing, hence if we apply theorem 6.3 to $\alpha(M)$, we find $C\left(\alpha\left(M_{g}\right)\right) \leq 2 g^{\prime}-2$. The sequence of inequalities:

$$
2 g^{\prime}-2 \leq \mathrm{HD}(\alpha(X)) \leq \underline{C}(\alpha(X)) \leq C(\alpha(X)) \leq C\left(\alpha\left(M_{g}\right)\right) \leq 2 g^{\prime}-2
$$

finishes the proof.

This work was supported in part by NSF Grant \#DMS-8610730(1).

\section{REFERENCES}

[AY] P. Arnoux \& J. C. Yoccoz. Construction de difféomorphismes pseudo-Anosov. C. R. Acad. Sci. Paris Sér. I Math. 292 (1981), 75-78.

[BO] R. Bowen. Markov partitions are not smooth. Proc. Amer. Math. Soc. 71 (1978), 130-132.

[DO] A. Douady \& J. Oesterlé. Dimension de Hausdorff des attracteurs. C. R. Acad. Sci. Paris Sér. I Math. 290 (1980), 1135-1138.

[Fra1] J. Franks. Anosov diffeomorphisms. Proc. Sympos. Pure Math. 14, 61-93.

[Fra2] J. Franks. Invariant sets of hyperbolic toral automorphisms. Amer. J. Math. 99 (1977), 1089-1095.

[Fri] D. Fried. Growth rate of surface homeomorphisms and flow equivalence. Ergod. Th. Dynam. Sys. 5 (1985), 539-563.

[Ha] S. Hancock. Construction of invariant sets for Anosov diffeomorphisms. J. London Math. Soc. 18 (1978), 339-348.

[Hi] M. Hirsch. On invariant subsets of hyperbolic sets. In Essays in topology and related topics, 1970, pp. $126-146$.

[Ir1] M. Irwin. The orbit of a Hölder continuous path under a hyperbolic toral automorphism. Ergod. Th. Dynam. Sys. 3 (1983), 345-349.

[Ir2] M. Irwin. Hölder continuous paths and hyperbolic toral automorphisms. Ergod. Th. Dynam. Sys. 6 (1986), 241-247.

[LY] F. Ledrappier \& L. S. Young. The metric entropy of diffeomorphisms part II: Relations between entropy, exponents and dimension. Ann. Math. 122 (1985), 540-574.

[Le1] G. Levitt. Propriètés homologiques des feuilletages des surfaces. C. R. Acad. Sci. Paris Sér. I Math. 293 (1981), 597-600.

[Le2] G. Levitt. Feuilletages des surfaces. Thèse d'État, Université Paris 7, 1983.

[Ma1] R. Mañé. Orbits of paths under hyperbolic toral automorphisms. Proc. Amer. Math. Soc. 73 (1979), 121-125.

[Ma2] R. Mañé. Invariant sets of Anosov diffeomorphisms. Invent. Math. 46 (1978), 147-152.

[Pr] F. Przytycki. Construction of invariant sets for Anosov diffeomorphisms and hyperbolic attractors. Studia Math. 58 (1980), 199-213.

[S] M. Shub. Alexander cocycles and dynamics. Astérisque 81 (1977), 395-414.

[Ur] M. Urbański. On the capacity of a continuum with a non dense orbit under a hyperbolic toral map, Studia Math. 81 (1985), 37-51. 